\title{
Assessment of the Predictive Validity of Etiologic Stroke Classification
}

E. Murat Arsava, MD; Johanna Helenius, MD; Ross Avery, BS; Mine H. Sorgun, MD; Gyeong-Moon Kim, MD, PhD; Octavio M. Pontes-Neto, MD, PhD; Kwang Yeol Park, MD; Jonathan Rosand, MD, MSc; Mark Vangel, PhD; Hakan Ay, MD

IMPORTANCE The ability of present-day etiologic stroke classification systems to generate subtypes with discrete stroke characteristics is not known.

OBJECTIVE To test the hypothesis that etiologic stroke subtyping identifies different disease processes that can be recognized through their different clinical courses.

DESIGN, SETTING, AND PARTICIPANTS We performed a head-to-head evaluation of the ability of the Causative Classification of Stroke (CCS), Trial of Org 10172 in Acute Stroke Treatment (TOAST), and ASCO (A for atherosclerosis, $\mathrm{S}$ for small-vessel disease, $\mathrm{C}$ for cardiac source, and O for other cause) classification systems to generate etiologic subtypes with different clinical, imaging, and prognostic characteristics in 1816 patients with ischemic stroke. This study included 2 cohorts recruited at separate periods; the first cohort was recruited between April 2003 and June 2006 and the second between June 2009 and December 2011. Data analysis was performed between June 2014 and May 2016.

MAIN OUTCOMES AND MEASURES Separate teams of stroke-trained neurologists performed CCS, TOAST, and ASCO classifications based on information available at the time of hospital discharge. We assessed the association between etiologic subtypes and stroke characteristics by computing receiver operating characteristic curves for binary variables (90-day stroke recurrence and 90 -day mortality) and by calculating the ratio of between-category to within-category variability from the analysis of variance for continuous variables (admission National Institutes of Health Stroke Scale score and acute infarct volume).

RESULTS Among the 1816 patients included, the median age was 70 years (interquartile range, $58-80$ years) ( 830 women [46\%]). The classification systems differed in their ability to assign stroke etiologies into known subtypes; the size of the undetermined category was $33 \%$ by CCS, $53 \%$ by TOAST, and $42 \%$ by ASCO ( $P<.001$ for all binary comparisons). All systems provided significant discrimination for the validation variables tested. For the primary validation variable (90-day recurrence), the area under the receiver operating characteristic curve was $0.71(95 \% \mathrm{Cl}, 0.66-0.75)$ for CCS, $0.61(95 \% \mathrm{Cl}, 0.56-0.67)$ for TOAST, and $0.66(95 \% \mathrm{Cl}, 0.60-0.71)$ for ASCO $(P=.01$ for CCS vs ASCO; $P<.001$ for CCS vs TOAST; $P=.13$ for ASCO vs TOAST). The classification systems exhibited similar discrimination for 90 -day mortality. For admission National Institutes of Health Stroke Scale score and acute infarct volume, CCS generated more distinct subtypes with higher between-category to within-category variability than TOAST and ASCO.

CONCLUSIONS AND RELEVANCE Our findings suggest that the major etiologic stroke subtypes are distinct categories with different stroke characteristics irrespective of the classification system used to identify them. We further show that CCS generates discrete etiologic categories with more diverse clinical, imaging, and prognostic characteristics than either TOAST or ASCO.
JAMA Neurol. 2017;74(4):419-426. doi:10.1001/jamaneurol.2016.5815 Published online February 27, 2017
Editorial page 388

Supplemental content
Author Affiliations: Author affiliations are listed at the end of this article.

Corresponding Author: Hakan Ay, MD, AA Martinos Center for Biomedical Imaging and Stroke Service, Departments of Radiology and Neurology, Massachusetts General Hospital, Harvard Medical School, 149 13th St, Room 2301 Charlestown, MA 02129 (hay@mgh.harvard.edu). 
T he quality of etiologic classification depends on the ability to generate homogenous subtypes with discrete outcomes (discriminative validity), the clarity of classification rules to ensure utility at different settings by different investigators (reliability), and the capacity to segregate etiologies into known subtypes rather than grouping them into a heterogeneous "undetermined" category. ${ }^{1}$ The cost of diagnostic investigations to identify the underlying etiology is estimated to be $\$ 7.7$ billion annually in the United States. ${ }^{2,3}$ Despite this high cost to the public, little is known about the validity of etiologic subtype information. Previous studies of stroke classification have primarily focused on reliability. ${ }^{4-10}$ Published reports on validity are limited by small sample sizes, variable definitions for subtypes and outcome events, and the use of outdated classification and diagnostic evaluation algorithms. ${ }^{11-13}$ In the present study, we sought to determine the predictive or discriminative validity of etiologic classification by assessing the capacity of 3 novel classification systems-the automated Causative Classification of Stroke (CCS) system, ${ }^{5}$ the Trial of Org 10172 in Acute Stroke Treatment (TOAST) algorithm, ${ }^{6}$ and ASCO (A for atherosclerosis, $S$ for small-vessel disease, $C$ for cardiac source, and O for other cause) system ${ }^{14,15}$-to unequivocally generate subtypes with distinct stroke features in a cohort of patients with acute ischemic stroke who underwent comprehensive diagnostic evaluation.

\section{Methods}

\section{Study Population and Data Collection}

The study population consisted of 2 separate cohorts with magnetic resonance imaging-confirmed ischemic stroke admitted to Massachusetts General Hospital during different periods. Cohort 1 was recruited between April 2003 and June 2006 as a part of an institutional registry project. ${ }^{16}$ Cohort 2 was recruited between June 2009 and December 2011 within the context of a National Institutes of Health (NIH)-funded study (Heart Brain Interactions in Human Acute Ischemic Stroke Study).${ }^{17}$ Both cohorts were consecutive and excluded those who were admitted after 72 hours of symptom onset. We collected pertinent clinical, imaging, and laboratory data in each patient through the review of electronic medical records at the time of hospital discharge (or in-hospital death) using a standard data collection manual. We generated a stroke classification form that included demographic characteristics, presenting symptoms and clinical stroke features, stroke risk factors, and diagnostic test findings. These forms were later used by adjudicators to determine etiologic stroke subtypes. Data analysis was performed between June 2014 and May 2016. The study was approved by the Partners Institutional Review Board; written or oral consent was obtained from patients.

\section{Etiologic Classification}

We identified etiologic stroke subtypes using CCS, TOAST, and ASCO systems. The CCS system is a web-based, semiautomated, probabilistic decision-support algorithm that integrates clinical, imaging, and laboratory information from the

\section{Key Points}

Question Can etiologic stroke subtyping generate categories with discrete clinical, imaging, and prognostic characteristics?

Findings A head-to-head, blind evaluation of Causative Classification of Stroke, Trial of Org 10172 in Acute Stroke Treatment, and ASCO (A for atherosclerosis, S for small-vessel disease, $\mathrm{C}$ for cardiac source, and $\mathrm{O}$ for other cause) classification systems in 1816 consecutive patients with ischemic stroke revealed that all systems generated etiologic subtypes with different 90-day stroke recurrence, 90-day survival, admission stroke severity, and acute infarct burden. The Causative Classification of Stroke system redistributed $20 \%$ to $40 \%$ of the population assigned into the undetermined category by other systems into known subtypes and provided a greater discrimination for most of the stroke characteristics tested as compared with the Trial of Org 10172 in Acute Stroke Treatment and ASCO systems.

Meaning Etiologic stroke classification identifies discrete categories with different stroke features.

stroke workup using evidence-based criteria and identifies the most likely causative etiology. ${ }^{5}$ The TOAST system provides causative etiologic subtypes based on clinical impression and diagnostic test findings. ${ }^{6}$ The ASCO system is a phenotypic algorithm that assigns stroke subtypes into nonmutually exclusive categories without weighting toward the most likely cause in the presence of multiple competing etiologies. ${ }^{14,15}$ All classification methods stratify stroke subtypes into the same 5 major categories: large-artery atherosclerosis (LAA) causing 50\% or more stenosis, cardiac embolism (CE), small-artery occlusion (SAO), uncommon causes, and undetermined causes. To compare phenotypic ASCO and causative CCS and TOAST, we converted ASCO from phenotypic to causative as previously described. ${ }^{18}$ Here, we considered only grade 1 evidence when determining ASCO subtypes. To further ensure consistency between ASCO grade 1 and the other 2 systems, we reassigned low- or uncertain-risk cardiac sources into the undetermined category in CSS and TOAST. ${ }^{14}$

Separate teams of stroke-trained neurologists performed subtype classifications for each classification system. As a part of the training process, the senior author (H.A.) reassessed the initial 20 cases classified by each adjudicator and provided feedback. Adjudicators performed subtype determinations using clinical and diagnostic information available at the time of discharge through inspection of the stroke classification form for each case. Subtype adjudicators remained blinded to each other's subtype assignments and validation end points for the duration of the study. The CCS algorithm was developed at the Massachusetts General Hospital. None of the adjudicators who performed CCS classifications were involved in its design or development.

\section{Validation End Point Assessment}

Because there is no ground truth in etiologic classification against which the accuracy of classifications can be tested, we assessed validity by studying the ability of etiologic subtypes to discriminate between important stroke characteristics. Our 
objective was to determine whether etiologic classification could serve its purpose: ie, whether the classification systems could characterize and capture clinical characteristics of interest in relation to different etiologic subtypes. This approach is an established method used to determine the predictive or discriminative validity of diagnostic instruments and is frequently used in social sciences and in medicine when there is no unequivocal reference standard (such as for diagnosis of psychiatric disorders). ${ }^{19}$ The primary goal of diagnostic stroke evaluation is to identify the underlying etiology so that targeted treatments to prevent a second stroke can be instituted. For this reason, we chose 90-day stroke recurrence as the primary validation end point for quality assessment. We used 90 days as a cutoff because approximately half of the recurrent strokes observed over the course of a year occur within the first 90 days. ${ }^{20,21}$ Other validation end points were 90 day survival, admission National Institutes of Health Stroke Scale (NIHSS) score, and admission infarct volume. These features are frequently used for patient selection and prognostication in clinical stroke research and, hence, are relevant for validity assessment.

Follow-up evaluation included assessment of survival and recurrent stroke status at 90 days, with a range of 75 to 105 days. One investigator blinded to the etiologic subtype results performed outcome assessments through in-person evaluations and through telephone interviews or through reviews of physician notes obtained during outpatient visits when the patient was unavailable for a follow-up visit. Physician notes provided indepth descriptions of the timing, clinical features, and diagnostic evaluation findings of the recurrent stroke. In addition, we used the Social Security Death Index to confirm the survival status at 90 days. We defined recurrent stroke as a clinical incident that was clearly attributable to a new area of brain infarct, which could be observed with imaging as a lesion spatially distinct from the index infarct. ${ }^{16}$ Two investigators (J.H. and M.H.S.) blinded to etiologic subtype information calculated index infarct volume by manually outlining acute infarcts on diffusionweighted images acquired at the time of hospital admission using semiautomated software (MRIcro software, University of Nottingham, Nottingham, England).

\section{Diagnostic Investigations and Preventive Treatments}

All patients underwent a thorough diagnostic evaluation to identify the underlying etiology. The standard investigation battery included brain imaging (computed tomography and/or magnetic resonance imaging), extracranial and intracranial vascular imaging, electrocardiography, and transthoracic echocardiography. Specific blood and cerebrospinal fluid tests, transoesophageal echocardiography, and long-term cardiac rhythm monitoring were performed when there was no source on other investigations or when the patient was younger than 55 years old. All patients received preventive treatment as recommended by the secondary stroke prevention guidelines. ${ }^{22}$ These included risk factor modification and control, anticoagulant therapy for major cardiac sources, carotid revascularization and antiplatelet treatment for LAA, antiplatelet treatment for minor cardiac sources, cryptogenic stroke, SAO, and disease-specific treatment for uncommon causes of stroke.

\section{Statistical Analysis}

We assessed the relationship between etiologic categories and baseline clinical and imaging variables using the KruskalWallis test for continuous variables and the Fisher exact test or the $\chi^{2}$ test for categorical variables. We used the $\chi^{2}$ test to compare the size of etiologic categories by CCS and TOAST. We calculated agreement between classification algorithms in etiologic subtype assignments using unweighted $\kappa$ statistics. ${ }^{23}$

We constructed the Cox proportional hazard regression models that included time to outcome as response and etiologic subtypes as independent variables. We determined the cumulative risk of binary end points using Kaplan-Meier analysis. We used the log-rank test to examine the relationship between etiologic subtypes and cumulative outcome rates. We further constructed multivariable Cox regression models to examine the relationship between etiologic subtypes and primary outcome adjusting for baseline covariates with a univariate $P<.05$. We computed receiver operating characteristic curves and calculated the area under the curve (AUC) to assess the discriminative ability of etiologic subtypes for binary end points. We compared the AUC values for each algorithm using the $z$ test. $^{24}$

For continuous validation variables (admission NIHSS score and acute infarct volume), we used the between-category $F$ statistic to determine each algorithm's capacity to generate subtypes with discrete features. The better classifier had a higher ratio of between-category to within-category variability, and hence a larger $F$ statistic. ${ }^{25}$ Numerical variables were expressed as median (interquartile range) or mean (95\% CI). $P<.05$ was considered statistically significant. All statistical analyses were performed using SPSS version 16 (IBM).

\section{Results}

The study included a total of 2284 patients with acute ischemic stroke. We excluded 468 patients (20\%) who were not available for follow-up owing to relocation or refusal to participate. There was no difference in patient characteristics between cohorts with and without follow-up assessment (eTable 1 in the Supplement). Of the remaining 1816 patients, 998 were in cohort 1 and 818 in cohort 2 . The study cohorts were similar with the exception that a greater percentage of the patients received statin treatment while fewer patients received anticoagulant therapy and underwent carotid revascularization in cohort 2 (eTable 2 in the Supplement).

Baseline characteristics of the study cohort are presented in Table 1. Classification systems differed in their ability to assign stroke etiologies into known subtypes, as opposed to the undetermined category (Table 2); the size of undetermined category was $33 \%$ by CCS, $53 \%$ by TOAST, and $42 \%$ by ASCO $(P<.001$ for CCS vs TOAST, CCS vs ASCO, and ASCO vs TOAST). The CCS-undetermined category was comprised of 9\% cryptogenic, $21 \%$ minor CE, $1 \%$ incomplete evaluation, and $2 \%$ multiple competing etiologies. The corresponding proportions for TOAST-undetermined categories were $11 \%, 19 \%, 1 \%$, and $22 \%$, respectively. The undetermined category in ASCO consisted of $37 \%$ cryptogenic and $5 \%$ multiple competing etiologies. The 
$\kappa$ value for agreement was 0.70 (95\% CI, 0.68-0.73) between CCS and TOAST, 0.85 (95\% CI, 0.83-0.87) between CCS and ASCO, and 0.72 (95\% CI, 0.69-0.74) between TOAST and ASCO.

Recurrent ischemic stroke occurred in 100 patients. The cumulative recurrence rate was 6.0\% (95\% CI, 4.8\%-7.2\%). Recurrence-free survival varied by etiologic subtype (log-rank test; $P<.001$ for all systems; Figure 1). The CCS system demonstrated better separation in Kaplan-Meier curves compared with TOAST and ASCO ( $\chi^{2}, 53.2$ for CCS, 25.1 for TOAST, and 32.7 for ASCO). The AUC for 90-day stroke recurrence was 0.71 (95\% CI, 0.66-0.75) for CCS, 0.61 (95\% CI, 0.56-0.67) for TOAST, and 0.66 (95\% CI, 0.60-0.71) for ASCO $(P<.001$ for CCS vs TOAST; $P=.01$

\begin{tabular}{|c|c|}
\hline & No. (\%) \\
\hline Characteristic & $\begin{array}{l}\text { Study Population } \\
(\mathrm{n}=1816)\end{array}$ \\
\hline Age, median, (IQR), y & $70(58-80)$ \\
\hline Women & $830(46)$ \\
\hline \multicolumn{2}{|l|}{ Risk factors } \\
\hline Hypertension & 1250 (69) \\
\hline Diabetes & $428(24)$ \\
\hline Coronary artery disease & $364(20)$ \\
\hline Atrial fibrillation & $467(26)$ \\
\hline Smoking & $340(19)$ \\
\hline \multicolumn{2}{|l|}{ Diagnostic evaluation } \\
\hline Brain imaging & $1816(100)$ \\
\hline CT or MR angiography & $1707(94)$ \\
\hline ECG & $1816(100)$ \\
\hline Transthoracic/transesophageal echocardiography & $1653(91)$ \\
\hline Holter monitoring & $1090(60)$ \\
\hline \multicolumn{2}{|l|}{ Secondary prevention } \\
\hline Anticoagulant treatment & $655(36)$ \\
\hline Antiaggregant treatment & $1238(68)$ \\
\hline Statin treatment & $1180(65)$ \\
\hline Carotid revascularization & $102(6)$ \\
\hline Admission NIHSS score, median (IQR) & $4(2-11)$ \\
\hline Admission DWI lesion volume, median (IQR), $\mathrm{mL}$ & $5.6(1.3-26.2)$ \\
\hline 90-d Cumulative recurrence rate, $\%(95 \% \mathrm{Cl})$ & $6.0(4.8-7.2)$ \\
\hline 90-d Cumulative mortality rate, $\%(95 \% \mathrm{Cl})$ & $16.1(14.3-17.9)$ \\
\hline
\end{tabular}

Abbreviations: CT, computed tomography; DWI, diffusion-weighted image; ECG, electrocardiography; IQR, interquartile range; MR, magnetic resonance; NIHSS, National Institutes of Health Stroke Scale. for CCS vs ASCO; and $P=.13$ for TOAST vs ASCO; Figure 2A). The hazard ratio for stroke recurrence varied by up to 2 -folds across the subtypes between the classification systems (Table 3). A time epoch analysis showed that the cumulative recurrence rate did not differ between cohort 1 and cohort 2 (6.6\% vs 5.3\%; $P=.29$ ). Classification systems exhibited similar discrimination for $90-$ day recurrence when tested separately in cohort 1 and cohort 2 (eTable 2 in the Supplement). The relationship between etiologic subtypes and 90-day stroke recurrence did not change after adjusting for variables with a univariate $P<.05$ (hypertension and admission NIHSS score). Adding the type of preventive treatment (anticoagulation, antiplatelet, statin, or carotid intervention) into the regression models did not alter the relationship between stroke subtypes and 90-day recurrence (eTable 3 in the Supplement). The AUCs for CCS and TOAST were 0.68(95\% CI, 0.63-0.73) and 0.62 (95\% CIs 0.57-0.68), respectively ( $P=.03$ ) when data were analyzed after redistributing minor cardiac sources into the CE category in these 2 systems. Consideration of grade 1 evidence did not allow for redistribution of minor cardiac sources in ASCO.

A total of 290 patients died during the study period. The cumulative 90 -day mortality rate was $16.1 \%$ (95\% CI, 14.3\%$17.9 \%$ ). Survival varied by etiologic subtypes (log-rank test; $P<$.001 for all systems; Figure 1 and Table 3). All 3 classification systems provided similar discrimination for 90-day mortality; there was no difference in AUCs among CCS, TOAST, and ASCO (Figure 2B). Admission NIHSS score and acute infarct volume also differed by etiologic stroke subtypes (Table 3 ). The analysis of variance demonstrated that CCS generated more discrete and homogenous subtypes than TOAST and ASCO; the $F$ values were 39.78 for CCS, 34.99 for TOAST, and 33.21 for ASCO for NIHSS score and 11.21 for CCS, 8.84 for TOAST, and 9.07 for ASCO for infarct volume.

\section{Discussion}

This study assessed the validity of etiologic stroke classification by examining the strength of association between etiologic stroke subtypes and important stroke characteristics in a consecutive series of patients who underwent a thorough and standard diagnostic assessment for stroke etiology. The major finding of this study is that 90-day stroke recurrence, 90day survival, admission stroke severity, and admission infarct volume differ by etiologic stroke subtypes regardless of

\begin{tabular}{|c|c|c|c|c|c|c|}
\hline \multirow[b]{2}{*}{ Subtype } & \multicolumn{3}{|l|}{ №. (\%) } & \multicolumn{3}{|l|}{$P$ Value } \\
\hline & CCS & TOAST & ASCO & CCS vs TOAST & CCS vs ASCO & TOAST vs ASCO \\
\hline Large-artery atherosclerosis & $395(22)$ & $197(11)$ & $353(19)$ & $<.001$ & .09 & $<.001$ \\
\hline Cardiac embolism & $443(24)$ & $441(24)$ & $449(25)$ & .92 & .82 & .75 \\
\hline Small-artery occlusion & $234(13)$ & $113(6)$ & $139(8)$ & $<.001$ & $<.001$ & .09 \\
\hline Uncommon causes & $149(8)$ & $113(6)$ & $105(6)$ & .02 & .004 & .58 \\
\hline Undetermined causes & $595(33)$ & $952(53)$ & $770(42)$ & $<.001$ & $<.001$ & $<.001$ \\
\hline
\end{tabular}

Abbreviations: ASCO, A for atherosclerosis, S for small-vessel disease, C for cardiac source, and O for other cause; CCS, Causative Classification of Stroke; TOAST, Trial of Org 10172 in Acute Stroke Treatment. 
Figure 1. Kaplan-Meier Survival Curves
A CCS 90-d recurrence

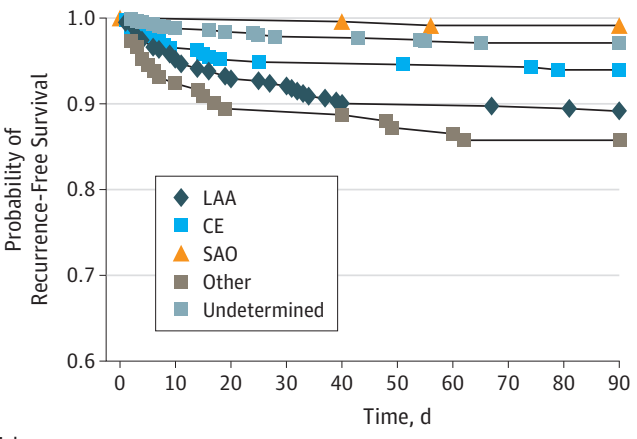

$\begin{array}{lllll}\begin{array}{l}\text { No. at risk } \\ \text { LAA }\end{array} & 395 & 316 & 306 & 298 \\ \text { CE } & 443 & 321 & 308 & 294 \\ \text { SAO } & 234 & 231 & 228 & 227 \\ \text { Other } & 149 & 122 & 118 & 115 \\ \text { Undetermined } & 595 & 524 & 511 & 500\end{array}$

C ASCO 90-d recurrence

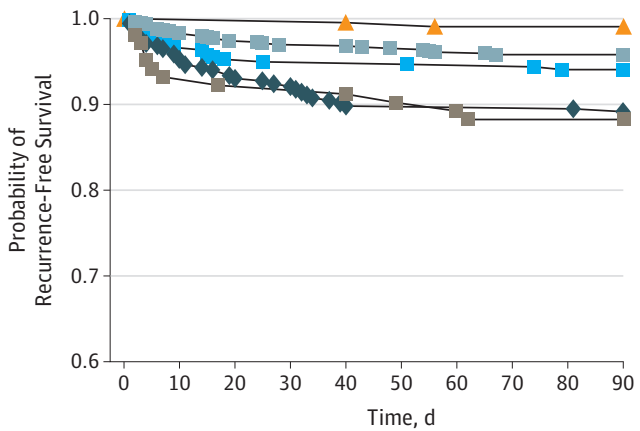

No. at risk

$\begin{array}{lcccc}\text { LAA } & 353 & 285 & 275 & 268 \\ \text { CE } & 449 & 327 & 314 & 300 \\ \text { SAO } & 139 & 138 & 137 & 137 \\ \text { Other } & 105 & 93 & 91 & 89 \\ \text { Undetermined } & 770 & 671 & 654 & 640\end{array}$

E TOAST 90-d survival

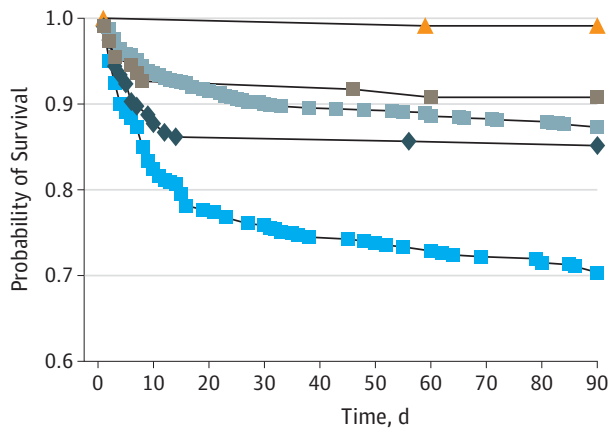

$\begin{array}{lllll}\begin{array}{l}\text { No. at risk } \\ \text { LAA }\end{array} & 197 & 170 & 169 & 168 \\ \text { CE } & 441 & 336 & 324 & 311 \\ \text { SAO } & 113 & 113 & 112 & 112 \\ \text { Other } & 113 & 105 & 104 & 103 \\ \text { Undetermined } & 952 & 859 & 848 & 832\end{array}$

Kaplan-Meier curves for 90-day recurrence-free survival and 90-day survival for Causative Classification of Stroke (CCS) (A and D), Trial of Org 10172 in Acute Stroke Treatment (TOAST) (B and E), and ASCO (A for atherosclerosis, S for small-vessel disease, $C$ for cardiac source, and $O$ for other cause) ( $C$ and $F$ ), showing that both outcomes vary by etiologic subtypes (log-rank test; $P<.001$
B TOAST 90-d recurrence
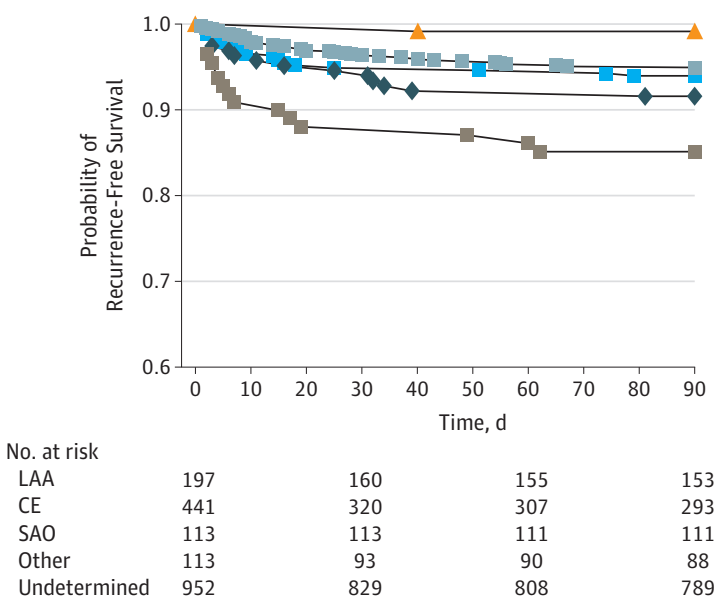

D CCS 90-d survival
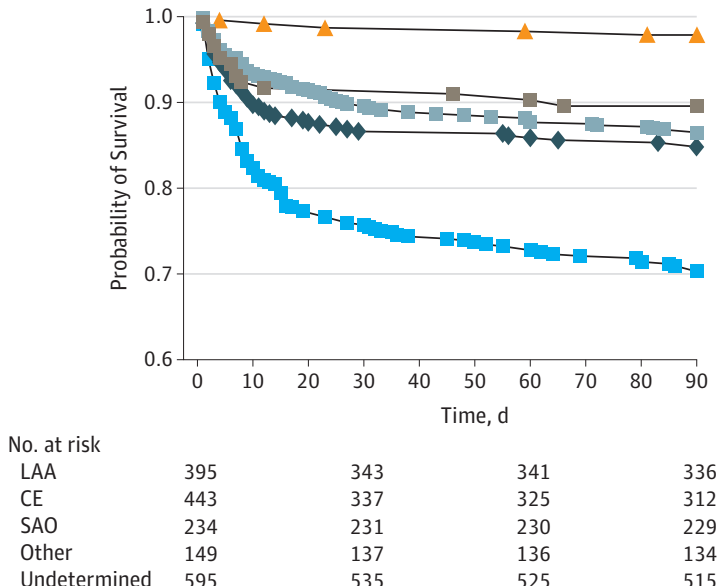

F ASCO 90-d survival

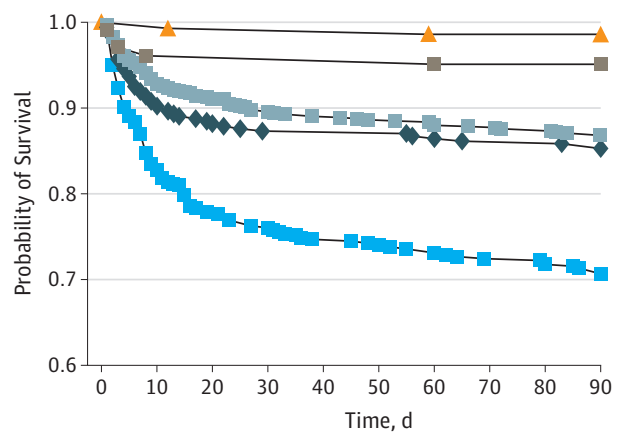

$\begin{array}{lllll}\begin{array}{l}\text { No. at risk } \\ \text { LAA }\end{array} & 353 & 309 & 307 & 302 \\ \text { CE } & 449 & 343 & 331 & 318 \\ \text { SAO } & 139 & 138 & 137 & 137 \\ \text { Other } & 105 & 101 & 101 & 100 \\ \text { Undetermined } & 770 & 692 & 681 & 669\end{array}$

for all systems). The CCS system demonstrates better separation in

Kaplan-Meier curves for recurrence-free survival compared with TOAST and ASCO ( $\chi^{2}, 53.2$ for CCS, 25.1 for TOAST, and 32.7 for ASCO). CE indicates cardiac embolism; LAA, large-artery atherosclerosis; and SAO, small-artery occlusion. 

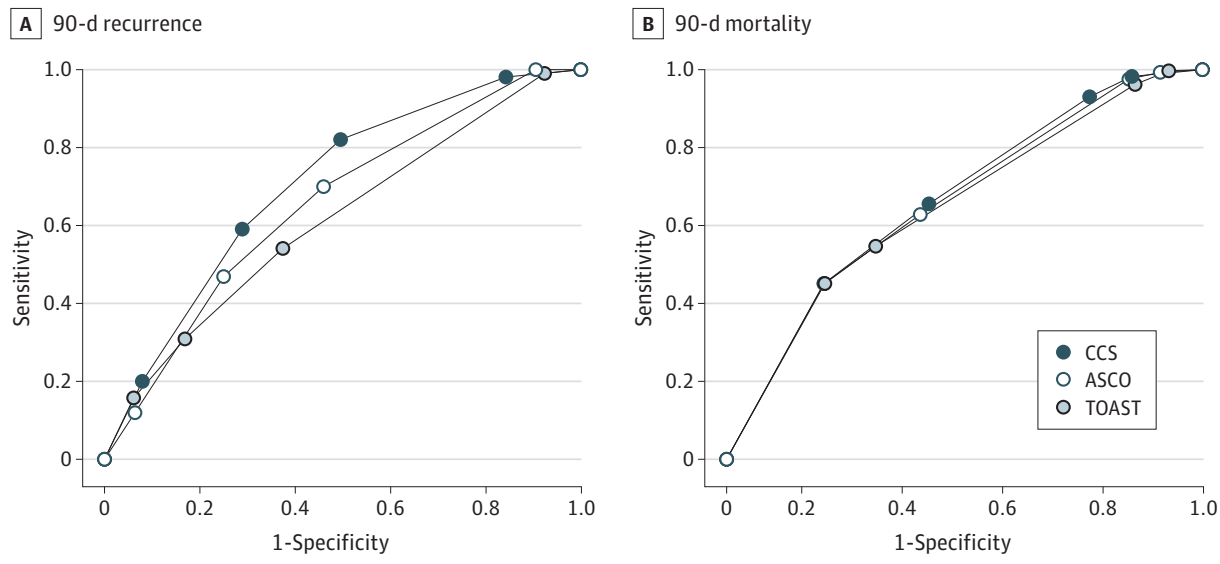

Receiver operating characteristic curves for discriminative ability of Causative Classification of Stroke (CCS), Trial of Org 10172 in Acute Stroke Treatment (TOAST), and ASCO (A for atherosclerosis, $\mathrm{S}$ for small-vessel disease, $\mathrm{C}$ for cardiac source, and $\mathrm{O}$ for other cause) for 90 -day recurrence-free survival (A) and 90-day survival (B). The area under the receiver operating characteristic

curves were 0.71 ( $95 \% \mathrm{Cl}, 0.66-0.75)$ for CCS, 0.61 ( $95 \% \mathrm{Cl}, 0.56-0.67)$ for TOAST, and $0.66(95 \% \mathrm{Cl}, 0.60-0.71)$ for ASCO for 90-day recurrence-free survival and 0.67 (95\% Cl, 0.64-0.70) for CCS, 0.65 (95\% Cl, 0.62-0.69) for TOAST, and 0.66 ( $95 \% \mathrm{Cl}, 0.63-0.69)$ for ASCO for 90-day survival.

\begin{tabular}{|c|c|c|c|c|c|c|}
\hline Variable & $\begin{array}{l}\text { Large-Artery } \\
\text { Atherosclerosis }\end{array}$ & Cardiac Embolism & $\begin{array}{l}\text { Small-Artery } \\
\text { Occlusion }\end{array}$ & Uncommon Causes & $\begin{array}{l}\text { Undetermined } \\
\text { Causes }\end{array}$ & $P$ Value \\
\hline \multicolumn{7}{|l|}{ 90-d Recurrence } \\
\hline \multicolumn{7}{|l|}{ CCS } \\
\hline Cumulative risk $(95 \% \mathrm{Cl})$ & $10.9(7.8-14.0)$ & $6.0(3.6-8.4)$ & $0.9(0.0-2.1)$ & $14.2(8.3-20.1)$ & $2.9(1.5-4.3)$ & \multirow{2}{*}{$<.001$} \\
\hline Hazard ratio $(95 \% \mathrm{Cl})$ & $3.9(2.2-7.0)$ & $2.2(1.2-4.1)$ & $0.3(0.1-1.3)$ & $5.3(2.8-10.3)$ & 1 [Reference] & \\
\hline \multicolumn{7}{|l|}{ TOAST } \\
\hline Cumulative risk \% $(95 \% \mathrm{Cl})$ & $8.4(4.3-12.5)$ & $6.1(3.7-8.5)$ & $0.9(0.0-2.7)$ & $14.9(8.2-21.6)$ & $5.1(3.7-6.5)$ & \multirow{2}{*}{$<.001$} \\
\hline Hazard ratio $(95 \% \mathrm{Cl})$ & $1.7(1.0-3.1)$ & $1.2(0.8-2.1)$ & $0.2(0.0-1.2)$ & $3.2(1.8-5.7)$ & 1 [Reference] & \\
\hline \multicolumn{7}{|l|}{ ASCO } \\
\hline Cumulative risk \% (95\% Cl) & $10.9(7.6-14.2)$ & $5.9(3.5-8.3)$ & $0.0(0.0-2.2)$ & $11.8(5.5-18.1)$ & $4.2(2.6-5.8)$ & \multirow{2}{*}{$<.001$} \\
\hline Hazard ratio $(95 \% \mathrm{Cl})$ & $2.7(1.6-4.4)$ & $1.5(0.9-2.5)$ & $0(0-0)$ & $3.0(1.5-5.8)$ & 1 [Reference] & \\
\hline \multicolumn{7}{|c|}{ 90-d Cumulative Mortality Risk, \% (95\% Cl) } \\
\hline CCS & $15.2(11.7-18.7)$ & $29.7(25.4-34.0)$ & $2.1(0.3-3.9)$ & $10.4(5.5-15.3)$ & $13.5(10.8-16.2)$ & $<.001$ \\
\hline TOAST & $14.9(10.0-19.8)$ & $29.6(25.3-33.9)$ & $0.9(0.0-2.7)$ & $9.2(3.7-14.7)$ & $12.7(10.5-14.9)$ & $<.001$ \\
\hline ASCO & $14.7(11.0-18.4)$ & $29.3(25.0-33.6)$ & $1.4(1.0-3.4)$ & $4.9(0.8-9.0)$ & $13.2(10.8-15.6)$ & $<.001$ \\
\hline \multicolumn{7}{|c|}{ Admission NIHSS Score, Median (IQR) } \\
\hline CCS & $4(2-11)$ & $9(3-16)$ & $2(1-5)$ & $5(2-12)$ & $4(1-9)$ & $<.001$ \\
\hline TOAST & $5(1-12)$ & $9(3-16)$ & $2(1-4)$ & $5(2-13)$ & $4(1-9)$ & $<.001$ \\
\hline ASCO & $4(1-11)$ & $9(3-16)$ & $2(1-5)$ & $4(2-12)$ & $4(1-9)$ & $<.001$ \\
\hline \multicolumn{7}{|c|}{ Admission Acute Infarct Volume, Median (IQR) } \\
\hline CCS & $9.7(2.5-25.3)$ & $15.5(3.6-49.6)$ & $0.9(0.5-1.7)$ & $8.1(3.3-41.2)$ & $4.0(1.2-23.3)$ & $<.001$ \\
\hline TOAST & $10.9(2.9-34.0)$ & $16.0(3.9-48.2)$ & $0.9(0.4-1.7)$ & $8.1(2.7-65.7)$ & $3.3(1.0-18.0)$ & $<.001$ \\
\hline ASCO & $9.9(3.0-27.0)$ & $15.4(3.6-49.6)$ & $0.9(0.5-1.5)$ & $7.6(2.5-33.9)$ & $2.9(1.0-19.1)$ & $<.001$ \\
\hline \multicolumn{3}{|c|}{$\begin{array}{l}\text { Abbreviations: ASCO, A for atherosclerosis, S for small-vessel disease, C for } \\
\text { cardiac source, and O for other cause; CCS, Causative Classification of Stroke; }\end{array}$} & \multicolumn{4}{|c|}{$\begin{array}{l}\text { IQR, interquartile range; NIHSS, National Institutes of Health Stroke Scale; } \\
\text { TOAST, Trial of Org } 10172 \text { in Acute Stroke Treatment. }\end{array}$} \\
\hline
\end{tabular}

the classification system used to identify them. We have observed several notable clinical, imaging, and prognostic features associated with etiologic subtypes: LAA was associated with a high 90-day risk for recurrence (approximately 10\%) whereas the undetermined category posed a moderate risk (3\%-
$5 \%$ ) and SAO exhibited a low risk (approximately 1\%). Strokes that developed secondary to cardiac embolism were associated with larger infarcts, more severe clinical deficit, and lower 90-day survival compared with other subtypes. We also noted that the uncommon-causes category had a risk for 90-day re- 
currence comparable with that of strokes caused by LAA. The high risk in the uncommon-causes category was largely driven by acute nonatherosclerotic arteriopathies such as arterial dissection, active vasculitis, and iatrogenic factors. Overall, the ability of current classification algorithms to generate distinct subtypes with discrete features supports the predictive validity of etiologic subtype information. We note that our findings may underestimate the true association between etiologic subtypes and stroke characteristics because we tested the validity in a treated population where the type of preventive treatment used was based on presumed stroke etiology. Preventive treatments for high-risk etiologies (LAA and CE) generally offer larger absolute risk reductions than those for lowrisk etiologies (SAO and cryptogenic), resulting in reduced variability in subtype-specific risk for recurrence. ${ }^{22}$ Hence, the reported associations should be expected to be greater when tested in untreated or poorly treated populations.

The quality of a classification algorithm can be assessed by the degree to which it stratifies stroke etiologies into categories with discrete clinical, radiographic, and prognostic categories. All of the classification systems tested in this study accomplished this goal but they did so to different degrees. The CCS system exhibited a stronger discrimination for the primary validation end point: 90-day stroke recurrence. Although CCS, TOAST, and ASCO stratify stroke etiologies into the same 5 subtypes, they use different classification criteria to do this. The TOAST and ASCO systems largely rely on the presence or absence of an abnormality and categorize etiologic findings without weighting toward the most likely etiology in the presence of multiple competing etiologies. ${ }^{6,14}$ In contrast, CCS identifies the causative subtype by taking into account clinical and imaging stroke features that convey causal information. ${ }^{5}$ The results of this study demonstrate that consideration of the causal evidence in identifying the etiologic subtype of stroke enhances the validity of stroke classification. A recent report from the National Institute of Neurological Disorders and Stroke-Stroke Genetics Network further supports the validity of the CCS approach. ${ }^{26}$ In that study, investigators identified 2 novel loci: one associated with LAA and the other associated with SAO. That discovery was made possible by using the CCS, as none of the associated single-nucleotide polymorphisms reached significance when using TOAST subtyping in the discovery data set.

\section{Strengths and Limitations}

The present study had several strengths. We performed a headto-head comparison of widely used classification algorithms in a consecutive cohort by using a standard manual for data collection based on detailed diagnostic investigations, and by ensuring blind adjudication of stroke subtypes and validation variables.
Our study had certain limitations as well. Twenty percent of the study population was excluded because 90-day follow-up information was unavailable. However, it is unlikely that this caused a selection bias because baseline patient characteristics and stroke features were similar between populations with and without 90-day follow-up data. We studied a well-investigated cohort that received a targeted preventive treatment for the underlying etiology as recommended by the guidelines. Caution should be observed when generalizing the results of the present study to settings where practice deviates considerably from the guideline recommendations. In the present study, subtype adjudicators used abstracted data collected using a manual that provided documentation of clinical findings and diagnostic test results in a standard manner. This minimized ambiguities and inconsistencies in the source data and, hence, led to high agreement rates between the classification systems. High agreement might have reduced the differences in discrimination for stroke characteristics and outcomes by the classification systems. The TOAST and ASCO systems require classification of multiple competing etiologies into the undetermined category. Deviating from the published rules by assigning multiple competing etiologies into known subtypes based on an expert opinion could result in lower agreement rates between CCS and the other systems because personal opinion differs from one physician to another. ${ }^{7-9}$ Overall, we expect that the validity advantage of CCS over TOAST and ASCO would be more obvious in settings where the disagreement rates between the classification systems are higher than reported in the present study. ${ }^{27}$

\section{Conclusions}

Our findings have both clinical and research implications. The prognostic information conveyed by etiologic subtypes disclosed in this study underscores the importance of carefully identifying and properly classifying the underlying etiology in every patient with stroke. Valid and reliable etiologic subtype information can be used for patient selection into clinical trials, phenotyping in genetic and epidemiological studies, selection of preventive treatment, and assessment of treatment response and prognosis. The ability to generate homogenous subtypes with distinct features could enhance statistical power in future trials of preventive treatments. This, in turn, could reduce study costs, save time, and, more importantly, increase the chances for discovery. Overall, our findings contribute to the understanding of clinical and research significance of etiologic subtype information.

\section{ARTICLE INFORMATION}

Accepted for Publication: December 7, 2016.

Published Online: February 27, 2017 doi:10.1001/jamaneurol.2016.5815

Author Affiliations: AA Martinos Center for Biomedical Imaging, Department of Radiology, Massachusetts General Hospital, Harvard Medical
School, Boston (Arsava, Helenius, Avery, Sorgun, Kim, Pontes-Neto, Park, Ay); Department of Neurology, Hacettepe University, Ankara, Turkey (Arsava); Department of Neurology, University of Massachusetts Medical School, Worcester (Helenius); now with Department of Neurology, Beth Israel Deaconess Medical Center, Boston, Massachusetts (Helenius); Lewis Katz School of
Medicine, Temple University, Philadelphia, Pennsylvania (Avery); Department of Neurology, Faculty of Medicine, Ankara University, Ankara, Turkey (Sorgun); Department of Neurology, Samsung Medical Center, Sungkyunkwan University School of Medicine, Seoul, South Korea (Kim); Ribeirão Preto School of Medicine, University of São Paulo, São Paulo, Brazil (Pontes-Neto); Department 
of Neurology, Chung-Ang University College of Medicine, Seoul, South Korea (Park); Stroke Service, Department of Neurology, Massachusetts General Hospital, Harvard Medical School, Boston (Rosand, Ay); Center for Human Genetic Research, Massachusetts General Hospital, Harvard Medical School, Boston (Rosand); Biostatistics Center, Massachusetts General Hospital, Harvard Medical School, Boston (Vangel).

Author Contributions: Dr Ay had full access to all the data in the study and takes responsibility for the integrity of the data and the accuracy of the data analysis.

Concept and design: Ay.

Acquisition, analysis, or interpretation of data: All authors.

Drafting of the manuscript: Arsava, Ay. Critical revision of the manuscript for important intellectual content: Helenius, Avery, Sorgun, Kim, Pontes-Neto, Park, Rosand, Vangel, Ay. Statistical analysis: Arsava, Vangel, Ay. Obtained funding: Ay.

Administrative, technical, or material support: Ay. Supervision: Ay.

Conflict of Interest Disclosures: Dr Rosand was supported by grants R01-NS073344 and R24NSO92983 from the National Institutes of Health. Dr Ay was supported by grant R01-NS059710 from the National Institutes of Health. Drs Ay and Arsava contributed to the development of the Causative Classification of Stroke system. No other disclosures were reported.

\section{REFERENCES}

1. Ay H. Advances in the diagnosis of etiologic subtypes of ischemic stroke. Curr Neurol Neurosci Rep. 2010;10(1):14-20

2. Taylor TN, Davis PH, Torner JC, Holmes J, Meyer JW, Jacobson MF. Lifetime cost of stroke in the United States. Stroke. 1996;27(9):1459-1466.

3. Diringer MN, Edwards DF, Mattson DT, et al. Predictors of acute hospital costs for treatment of ischemic stroke in an academic center. Stroke. 1999; 30(4): 724-728.

4. Ay $H$, Furie $K L$, Singhal $A$, Smith WS, Sorensen AG, Koroshetz WJ. An evidence-based causative classification system for acute ischemic stroke. Ann Neurol. 2005;58(5):688-697.

5. Ay H, Benner T, Arsava EM, et al. A computerized algorithm for etiologic classification of ischemic stroke: the Causative Classification of Stroke System. Stroke. 2007;38(11):2979-2984.

6. Adams HP Jr, Bendixen BH, Kappelle LJ, et al. Classification of subtype of acute ischemic stroke: definitions for use in a multicenter clinical trial: TOAST: Trial of Org 10172 in Acute Stroke Treatment. Stroke. 1993;24(1):35-41.

7. Goldstein LB, Jones MR, Matchar DB, et al. Improving the reliability of stroke subgroup classification using the Trial of ORG 10172 in Acute Stroke Treatment (TOAST) criteria. Stroke. 2001;32 (5):1091-1098

8. Gordon DL, Bendixen BH, Adams HP Jr, Clarke W, Kappelle LJ, Woolson RF; The TOAST Investigators. Interphysician agreement in the diagnosis of subtypes of acute ischemic stroke: implications for clinical trials. Neurology. 1993;43 (5):1021-1027.

9. Meschia JF, Barrett KM, Chukwudelunzu F, et al; Siblings with Ischemic Stroke Study (SWISS) Investigators. Interobserver agreement in the Trial of Org 10172 in Acute Stroke Treatment classification of stroke based on retrospective medical record review. J Stroke Cerebrovasc Dis. 2006;15(6):266-272

10. Arsava EM, Ballabio E, Benner T, et al; International Stroke Genetics Consortium. The Causative Classification of Stroke System: an international reliability and optimization study. Neurology. 2010;75(14):1277-1284.

11. Kolominsky-Rabas PL, Weber M, Gefeller O, Neundoerfer B, Heuschmann PU. Epidemiology of ischemic stroke subtypes according to TOAST criteria: incidence, recurrence, and long-term survival in ischemic stroke subtypes: a population-based study. Stroke. 2001;32(12): 2735-2740.

12. Lovett JK, Coull AJ, Rothwell PM. Early risk of recurrence by subtype of ischemic stroke in population-based incidence studies. Neurology. 2004:62(4):569-573.

13. Grau AJ, Weimar C, Buggle F, et al. Risk factors outcome, and treatment in subtypes of ischemic stroke: the German stroke data bank. Stroke. 2001; 32(11):2559-2566.

14. Amarenco $P$, Bogousslavsky J, Caplan LR, Donnan GA, Hennerici MG. New approach to stroke subtyping: the A-S-C-O (phenotypic) classification of stroke. Cerebrovasc Dis. 2009;27(5):502-508.

15. Amarenco P, Bogousslavsky J, Caplan LR, Donnan GA, Wolf ME, Hennerici MG. The ASCOD phenotyping of ischemic stroke (updated ASCO phenotyping). Cerebrovasc Dis. 2013;36(1):1-5.

16. Ay H, Gungor L, Arsava EM, et al. A score to predict early risk of recurrence after ischemic stroke. Neurology. 2010;74(2):128-135.
17. Arsava EM, Kim GM, Oliveira-Filho J, et al. Prediction of early recurrence after acute ischemic stroke. JAMA Neurol. 2016;73(4):396-401.

18. Marnane M, Duggan CA, Sheehan OC, et al. Stroke subtype classification to mechanism-specific and undetermined categories by TOAST, A-S-C-O, and causative classification system: direct comparison in the North Dublin population stroke study. Stroke. 2010;41(8):1579-1586.

19. Rutjes AW, Reitsma JB, Coomarasamy A Khan KS, Bossuyt PM. Evaluation of diagnostic tests when there is no gold standard: a review of methods. Health Technol Assess. 2007;11(50):iii, ix-51. 20. Hillen $T$, Coshall C, Tilling K, Rudd AG, McGovern R, Wolfe CD; South London Stroke Register. Cause of stroke recurrence is multifactorial: patterns, risk factors, and outcomes of stroke recurrence in the South London Stroke Register. Stroke. 2003;34(6):1457-1463.

21. Hankey GJ, Jamrozik K, Broadhurst RJ, et al. Long-term risk of first recurrent stroke in the Perth Community Stroke Study. Stroke. 1998;29(12): 2491-2500.

22. Kernan WN, Ovbiagele B, Black HR, et al; American Heart Association Stroke Council, Council on Cardiovascular and Stroke Nursing, Council on Clinical Cardiology, and Council on Peripheral Vascular Disease. Guidelines for the prevention of stroke in patients with stroke and transient ischemic attack: a guideline for healthcare professionals from the American Heart Association/American Stroke Association. Stroke. 2014:45(7):2160-2236.

23. Fleiss JL. Measuring nominal scale agreement among many raters. Psychol Bull. 1971;76(5):378-382.

24. Hanley JA, McNeil BJ. A method of comparing the areas under receiver operating characteristic curves derived from the same cases. Radiology. 1983;148(3):839-843.

25. Lomax RG, Hahs-Vaughn D, eds. Statistical Concepts: A Second Course. 4th ed. New York, NY: Routledge, Taylor \& Francis Group; 2012.

26. NINDS Stroke Genetics Network (SiGN); International Stroke Genetics Consortium (ISGC). Loci associated with ischaemic stroke and its subtypes (SiGN): a genome-wide association study. Lancet Neurol. 2016;15(2):174-184.

27. Sim J, Wright C, eds. Research in Health Care: Concepts, Designs and Methods. Cheltenham, England: Stanley Thornes (Publishers) Ltd; 2000. 